Research Paper

\title{
Preclinical Targeting of Human Acute Myeloid Leukemia Using CD4-specific Chimeric Antigen Receptor (CAR) T Cells and NK Cells
}

\author{
Huda Salman ${ }^{\circledR *}$, Kevin G. Pinz ${ }^{2 *}$, Masayuki Wada 2, Xiao Shuai ${ }^{3}$, Lulu E. Yan 2, Jessica C. Petrov 1, Yupo \\ $\mathrm{Ma}{ }^{1,2}$ \\ 1. Department of Internal Medicine, Stony Brook Medicine, Stony Brook University Medical Center, Stony Brook, NY 11794, USA \\ 2. iCell Gene Therapeutics LLC, Research \& Development Division, Long Island High Technology Incubator, Stony Brook, NY 11790, USA \\ 3. Department of Hematology, West China hospital of Sichuan University, Chengdu, P.R. China \\ * These authors contributed equally to this manuscript \\ $\triangle$ Corresponding author: Huda Salman, email: huda.salman@stonybrookmedicine.edu \\ (c) The author(s). This is an open access article distributed under the terms of the Creative Commons Attribution License (https://creativecommons.org/licenses/by/4.0/). \\ See http://ivyspring.com/terms for full terms and conditions.
}

Received: 2018.08.02; Accepted: 2019.05.12; Published: 2019.07.23

\begin{abstract}
Acute myeloid leukemia (AML) is an aggressive malignancy lacking targeted therapy due to shared molecular and transcriptional circuits as well as phenotypic markers with normal hematopoietic stem cells (HSCs). Identifying leukemia specific markers expressed on AML or AML subtypes for therapeutic targeting is of exquisite clinical value. Here we show that CD4, a T lymphocytes membrane glycoprotein that interacts with major histocompatibility complex class II antigens and is also expressed in certain AML subsets but not on HSCs is a proper target for genetically engineered chimeric antigen receptor $T$ cells (CAR-T cells). Treatment with CD4 redirected CAR-T cell (CD4CAR) specifically eliminated CD4-expressing AML cell lines in vitro and exhibited a potent anti-leukemic effect in a systemic AML murine model in vivo. We also utilized natural killers as another vehicle for CAR engineered cells and this strategy similarly and robustly eliminated CD4expressing AML cells in vitro and had a potent in vivo anti-leukemic effect and was noted to have shorter in vivo persistence. Our data offer a proof of concept for immunotherapeutic targeting of $\mathrm{CD} 4$ as a strategy to treat $\mathrm{CD} 4$ expressing refractory AML as a bridge to stem cell transplant (SCT) in a first in human clinical trial.
\end{abstract}

Key words: NK cells; immunotherapy; T-cell malignancies; chimeric antigen receptors, AML

\section{Introduction}

Despite the unprecedented progress in understanding the genetic and molecular biology of acute myeloid leukemia (AML), this has not translated into significant advances in therapy and the improvements that have occurred are primarily the result of dose escalation of standard agents during induction and consolidation and improvements in supportive care. Patients deemed refractory to these approaches may not qualify for curative SCT because of persistent or residual disease.

The striking success of CD19-specific chimeric antigen receptor $\mathrm{T}$ cell (CAR-T) therapies against acute lymphoblastic leukemia has not yet been matched in AML 1. One major obstacle to targeting AML with CAR-Ts is that many myeloid antigens are expressed at similar levels on normal and malignant cells. Eliminating leukemic cells therefore may occur at the expense of normal myeloid tissue toxicity, including myeloid progenitor cells, resulting in an unacceptable "on target, off tumor" effect. Several preclinical studies have reported CARs targeting AML-associated antigens such as Lewis $\mathrm{Y}, \mathrm{CD} 33$, $\mathrm{CD} 44 \mathrm{vCD} 123$, and folate receptor $\beta$ (FR $\beta)^{2-9}$. Among these, Lewis Y, CD33, and CD123 have been used 
clinically but sustained complete responses have not yet been reported. Toxicities toward normal hematopoietic progenitor cells (HPCs) associated with the CD33 and CD123 CAR-T cell treatments have also been of particular concern.

CD4 is expressed in $65.0 \%$ and $78.3 \%$ in M4 and M5 subtypes of AML, respectively and in $30-40 \%$ of the other AML subtypes ${ }^{10}$. CD4 is not expressed on hematopoietic stem or progenitor cells, nor is it expressed on non-hematopoietic cells ${ }^{10,11}$. It has been shown that CD4 depletion utilizing monoclonal antibodies in clinical trials was well-tolerated and reversible. Observed toxicities offer some insight into safety, but cannot be fully extrapolated to CAR T cells 12-15. Our group obtained an FDA IND (No 17945) to initiate a CD4CAR phase1 clinical trial in CD4 positive $T$ cell hematological malignancies. This trial will report on the safety of CD4 redirected CAR cell therapy in humans.

While CAR-expressing $\mathrm{T}$ cells have entered successfully clinical trials, experience with CAR-engineered NK cells is mainly restricted to pre-clinical investigations and predominantly to NK cell lines. To date, pre-clinical data have been reported for CAR-modified primary human NK cells redirected against CD19, CD20, CD244, and HER2 as well as CAR-expressing NK-92 cells targeting a broader range of cancer antigens ${ }^{16-20}$. Natural killer (NK) cells may represent alternative cytotoxic effectors for CAR-driven cytolysis. Allogeneic NK cells are expected to induce an immune response and be rejected after a few days, and even autologous NK cells should disappear relatively rapidly from the circulation, owing to their limited lifespan.

Here we present CD4-directed CAR T cells and CAR NK cells as potent and specific approach to eradicate $\mathrm{CD} 4+$ myeloid malignant cells of AML in vitro and in mouse xenografts. Our preclinical data set the bases for utilizing CD4-directed CAR T cells and CAR NK cells as a novel and effective treatment for patients with refractory CD4 + AML to eliminate residual disease as a bridge to more definitive therapy with allogeneic SCT.

\section{Materials and Methods}

\section{Blood donors, primary leukemia cells, and cell lines}

DC4 + human primary AML samples and normal peripheral blood mononuclear cells (PBMCs) were obtained from residual samples using a protocol approved by the Institutional Review Board of Stony Brook University. THP-1, U937, TALL104, and NK-92 cell lines were obtained from ATCC (Manassas, VA, USA). MOLM-13 was obtained from AddexBio (San
Diego, CA, USA) T cells were cultured in filtered $\mathrm{T}$ cell media, defined as 50\% AIM V, 40\% RPMI 1640 and $10 \%$ FBS, with $1 \%$ Pen/Strep (all Gibco, Waltham, MA, USA) and supplemented with IL-2 (300 IU/mL; Peprotech, Rocky Hill, NJ, USA), unless otherwise specified. NK-92 cells were cultured in filtered NK cell media, defined as alpha-MEM without ribonucleosides and deoxyribonucleosides with $2 \mathrm{mM}$ L-glutamine, $1.5 \mathrm{~g} / \mathrm{L}$ sodium bicarbonate (Gibco), $12.5 \%$ heat-inactivated horse serum (Gibco), 12.5\% heat-inactivated FBS (Atlanta Biologicals, Atlanta GA, USA), 1\% Pen/Strep (Gibco), 0.2\% inositol (Sigma), $0.02 \%$ folic acid (Fisher), and $50 \mathrm{uM}$ beta-mercaptoethanol (Fisher), supplemented with IL-2 (300 IU/mL), unless otherwise specified. THP-1, U937, and MOLM-13 cell lines were cultured in RPMI, 10\% FBS, 1\% Pen/Strep (Gibco). TALL104 cells were cultured in IMDM adding $300 \mathrm{IU} / \mathrm{ml}$ recombinant human IL-2, $2.5 \mathrm{mg} / \mathrm{ml}$ human albumin, $0.5 \mathrm{mg} / \mathrm{ml}$ D-mannitol, and 20\% FBS.

\section{Co-Culture target cell ablation assays}

In the CAR T cell co-cultures, CD4CAR T cells or GFP T cells (control) were incubated with target cells at ratios of 2:1 and 5:1 (200,000 or 500,000 effector cells to 100,000 target cells, respectively) in $1 \mathrm{~mL}$ T-cell culture media without IL-2 for $24 \mathrm{~h}$. Target cells were THP-1, U937, and MOLM-13 cell lines (acute myeloid leukemia cell lines expressing CD4), and primary bone marrow cells from two patients with AML. All target cells were pre-stained with CMTMR (Life Technologies) to distinguish them from $\mathrm{T}$ cells during flow analysis. As a negative control, CMTMR-stained TALL104 cells, which do not express CD4, were also incubated with CD4CAR T cells and GFP T cells in the same ratios. After 24 hours of co-culture, cells were stained with mouse anti-human CD4-APC antibody (Tonbo, San Diego, CA, USA). For dose-dependent experiments, MOLM-13 cells were co-cultured with CAR T cells at lower ratios from 0.25:1 (25,000 effector cells to 100,000 target cells) to $5: 1$ with a sequential titer.

In the CAR NK cell co-culture experiment, target cells were labeled with CMTMR prior to incubation with CD4CAR NK cells or GFP NK cells (control) in IL-2 free media, and all cells were labeled with mouse anti-human CD4-APC after $24 \mathrm{~h}$ co-culture. Following this incubation, cells were washed, centrifuged, and re-suspended in $2 \%$ formalin for flow analysis.

All of the co-culture assays were performed in two independent experiments. Analysis of anti-leukemic activity was performed by comparing the residual amount of cells left in the CD4CAR T or NK cells treated samples with the GFP control cells treated samples, and data was presented as both the 
tumor lysis percentage and absolute cell counts. Analysis was performed using Kaluza software (Beckman Coulter, Brea, CA, USA).

\section{In vivo mouse xenogeneic model}

Two sets of NSG mice (NOD.Cg-Prkdcscid Il2 $\mathrm{rg}_{\mathrm{gm} 1 \mathrm{Wjl}} / \mathrm{SzJ}$ ) from the Jackson Laboratory (Bar Harbor, ME, USA) were used under a Stony Brook University IACUC-approved protocol. Mice were all male and between 9 and 12 weeks old. For each set, 16 mice were irradiated with a sublethal $(2.0 \mathrm{~Gy})$ dose of gamma irradiation and $24 \mathrm{~h}$ later they were intravenously injected with luciferase-expressing MOLM-13 cells via tail vein in order to form a measurable systemic leukemia. They were then randomly assigned to the treatment group or control

\section{A Schematic of CD4CAR Lentiviral Vector}

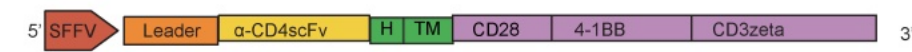

B CD4-3G CAR T cell Surface Expression

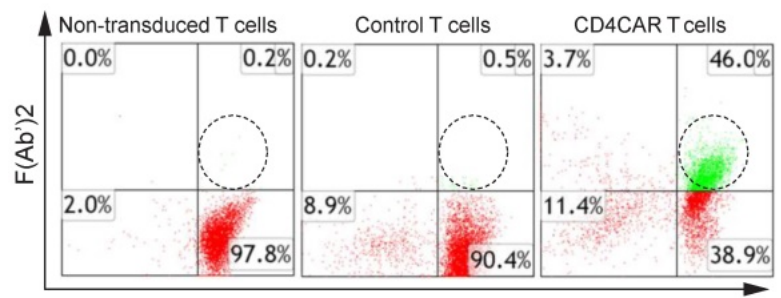

CD3

C

CD4-3G CAR NK cell Surface Expression

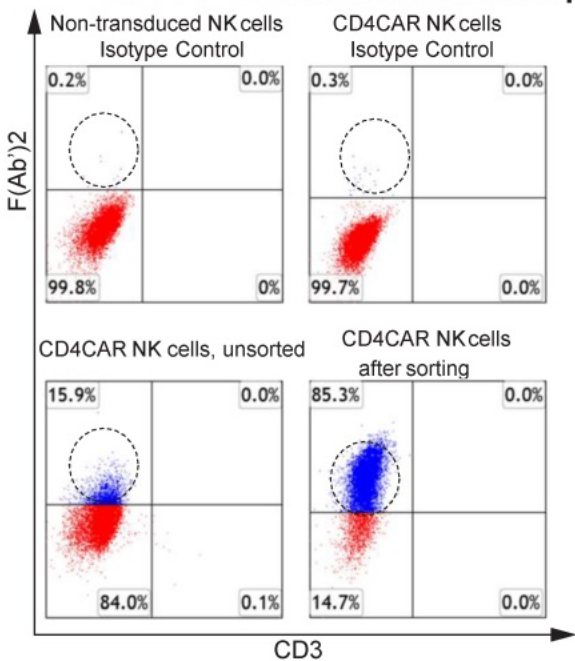

Figure 1. CD4CAR construct. (A) Schematic representation of the CD4CAR lentiviral vector (top). The CD4CAR construct is a tandem signaling domain that contains: a leader sequence; an anti-CD4scFv; a hinge domain (H); a transmembrane domain (TM); two co-stimulatory domains (CD28 and 4-1BB) that define the construct as a "third generation" CAR; and a CD3zeta intracellular signaling domain. (B) Flow cytometry analysis of CD4CAR expression on T-cell surface of non-transduced (left), GFP control (middle), and CD4CAR T-cells (right). Population in green delineates transduced CD4CAR T-cells. Gating was based off the vector and isotype controls.(C) Flow cytometry analysis of CD4CAR expression on NK-92 cell surface for non-transduced NK isotype control cells (upper left), CD4CAR NK isotype control cells (upper right), unsorted CD4CAR NK cells (lower left), and CD4CAR NK cells after sorting and expansion (lower right). Population in blue delineates transduced CD4CAR NK-92 cells. Gating was based off the vector and isotype controls. group. In the first set, mice were injected with $1.0 \times 10^{6}$ MOLM-13 cells and then given a course of $10 \times 10^{6}$ CD4CAR T cells $(n=8)$ or GFP control T cells $(n=8) 3$ days later via tail vein injection. In the second set, NSG mice were injected with $0.5 \times 10^{6}$ MOLM- 13 cells and then treated with a first dose of $5 \times 10^{6} \mathrm{CD} 4 \mathrm{CAR}$ NK cells $(n=8)$ or $5 \times 10^{6}$ GFP control NK cells $(n=8) 24$ hours later, and then a second dose of $5 \times 10^{6}$ CD4CAR NK cells or control cells on Day 10. After tumor cell inoculation, mice were monitored for systemic leukemia burden on the following sequential days (day 3, 6, 11 for the first set of mice; day 3, 7, 9 for the second set of mice). Mice were injected intraperitoneally (IP) with $100 \mu \mathrm{L}$ RediJect D-Luciferin (Perkin Elmer, Waltham, MA, USA) and subjected to IVIS imaging (PerkinElmer). Images were analyzed using Caliper Life Sciences software (PerkinElmer), and data was analyzed as previously described (Pinz 2015, Chen 2016).

Lentivirus production, CD4CAR transduction, and validation of C4CAR expression in both NK-92 and T cells, as well as statistical analysis are described in detail in Supplementary Information.

\section{Results}

\section{Generation of CD4CAR T cells}

The

CD4-specific

CAR (pRSC.SFFV.CD4.3G) was designed to contain an anti-CD4 scFv, a CD8-derived hinge $(\mathrm{H})$ and transmembrane (TM) domains, as well as intracellular CD28 and 4-1BB domains in tandem with the CD3zeta domain, defining the construct as a third-generation CAR (Figure 1A) (Pinz, 2015). Flow cytometry analysis showed that $\sim 46 \%$ of $\mathrm{T}$ cells expressed the $\mathrm{CD} 4 \mathrm{CAR} \mathrm{F}\left(\mathrm{Ab}^{\prime}\right)^{2}$ fragment after transduction (Figure 1B).

\section{CD4CAR T cells specifically eliminate CD4-expressing AML cell lines}

We first evaluated the anti-leukemia activity of CD4CAR $\mathrm{T}$ cells in individual co-culture killing assays with three CD4-positive acute myeloid leukemia cell lines: THP-1, U937, and MOLM-13. A CD4-negative T-ALL104 cell line was used as a negative control (Figure 2A). CD4CAR T cells were tested via 24hour co-cultures using the four cell lines with effector: target (E:T) cell ratios of 2:1 and 5:1. Compared to GFP control T cells, we observed that CD4CAR $\mathrm{T}$ cells specifically ablated CD4-positive populations (Figure 2A). At an E:T cell ratio of 2:1, we found that 
CD4CAR $\mathrm{T}$ cells already lysed over $74 \%$ of the MOLM-13 cells, and eliminated nearly $100 \%$ of the THP-1 and U937 cells. With an E: T ratio increase to $5: 1$, the lysis rate increased to $86 \%$ in the MOLM13 cells, remained nearly 100\% in the THP-1 and U937 cell lines. Conversely, CD4CAR T cells did not lyse the negative control T-ALL104 cells at either E: T ratio (Figure 2B).

In order to rule out the possibility that $\mathrm{T}$ cell expansion after antigen stimulation might confound the cytolysis rate, we also compared absolute cell counts to verify the CAR T cells cytotoxicity assay. We found that the absolute cell numbers of leukemic

A

CD4CAR T-cells + AML Leukemic Cell Line Co-Culture $\sim \mathrm{E}: \mathrm{T}=$ 2:1

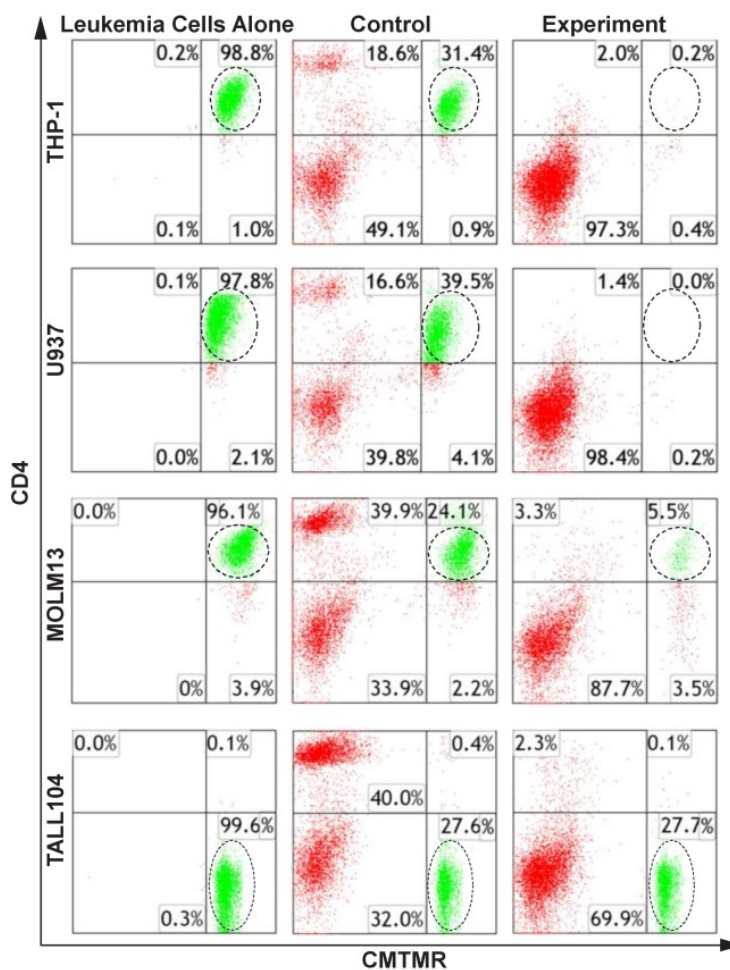

B

B AML Leukemic Cell Line Co-Cultures At an $E: T$ of $2: 1$ and 5:1

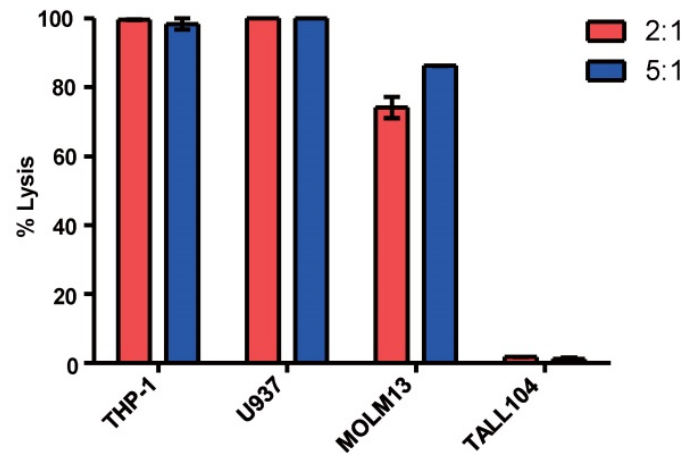

target cells THP-1, U937, and MOLM13 were significantly decreased in the CAR $\mathrm{T}$ cell treated group, compared with those in the GFP control group in the co-culture studies (Figure 2C).

Furthermore, we investigated the potential dose-dependent relationship of CD4CAR $\mathrm{T}$ cells by co-culturing MOLM-13 cells with sequential E: T dilution ratios of $0.25: 1,0.5: 1,1: 1,2: 1$, and 5:1. The result showed that target cell lysis rates were $1.3 \%$, $20 \%, 64.8 \%, 73 \%$, and $81 \%$, respectively (Figure S1), therefore demonstrating a strong anti-leukemia dose-dependent effect of CD4CAR T cells.

\section{Absolute Cell Counts of AML Cell \\ Line Co-Culture $\sim \mathrm{E}: \mathrm{T}=2: 1$}

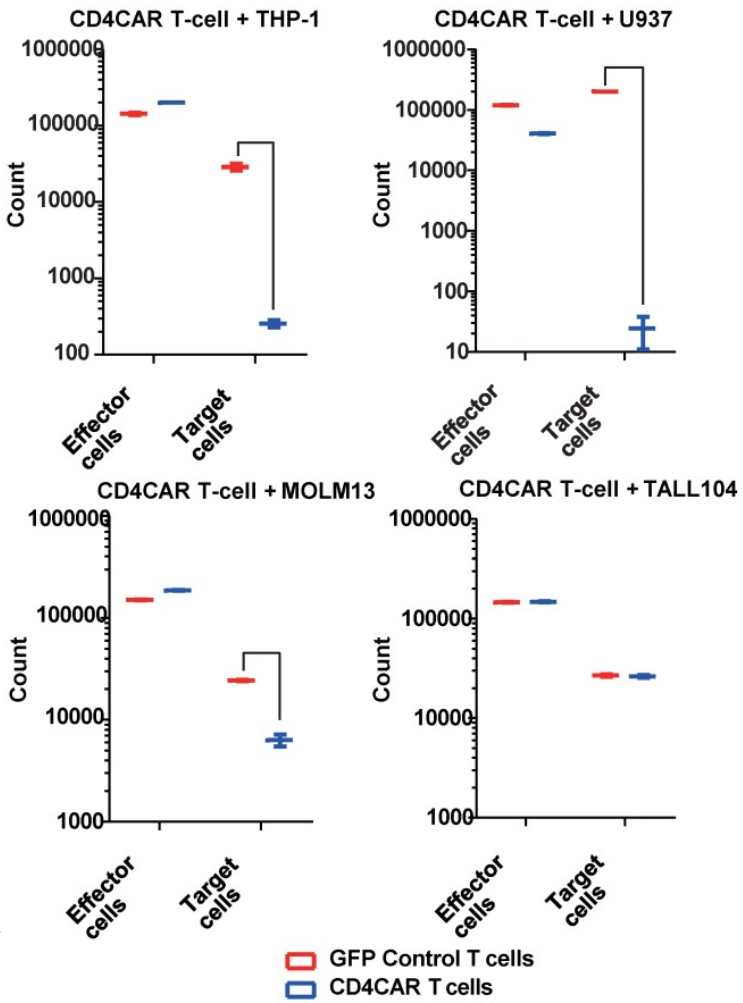

Figure 2: CD4CAR T-cells ablate CD4+ AML cell lines in vitro. (A) Co-culture experiments with CD4CAR T-cells were performed at E: T ratios of 2:1 and 5:1 for 24 hours. CD4+ target cell lines used are THP-1, U937, and MOLM13. TALL104 is a CD4- control. Target populations were quantified via flow cytometry using CD3 and pre-labeled CMTMR target cells to distinguish T-cell and target cell populations. Populations encircled highlight target cell lysis. (B) Graphical summary of CD4CAR T-cell in vitro assays against AML cell lines. Each bar represents the average percent cell lysis for duplicate samples; $N=2$ for all.(C) Absolute cell counts of target cell cultures with CD4CAR T-cells at an E: T ratio of 2:1. Control and CD4CAR treatment samples are labeled in red and blue respectively with effector and target cell counts performed via FACS analysis. 


\section{CD4CAR T cells specifically target and lyse CD4+ primary AML leukemic cells}

We next tested the efficiency of CD4CAR T cells in recognizing and killing primary AML leukemic cells. Co-culture experiments were conducted using clinical samples from two patients with CD4+ acute myeloid leukemia (PT1 and PT2). The two patients' bone marrow samples contained $59.3 \%$ and $49.7 \%$ of CD4-positive cells, respectively (Figure 3A). This corresponded with the blast population in the two patients' marrows (data not shown). In each case, a high level of target cell lysis was observed at either E: $\mathrm{T}$ ratio, as compared to GFP control T cells (Figure 3B). The potent cell lysis activity was also confirmed by absolute cell counting, with a significant reduction in residual target cells in the CD4CAR T cell-treated group (Figure $3 \mathrm{C}$ ), compared to the GFP control group. The results showed that CD4CAR $\mathrm{T}$ cells specifically targeted and eradicated primary myelogenous leukemic cells expressing CD4 in vitro.

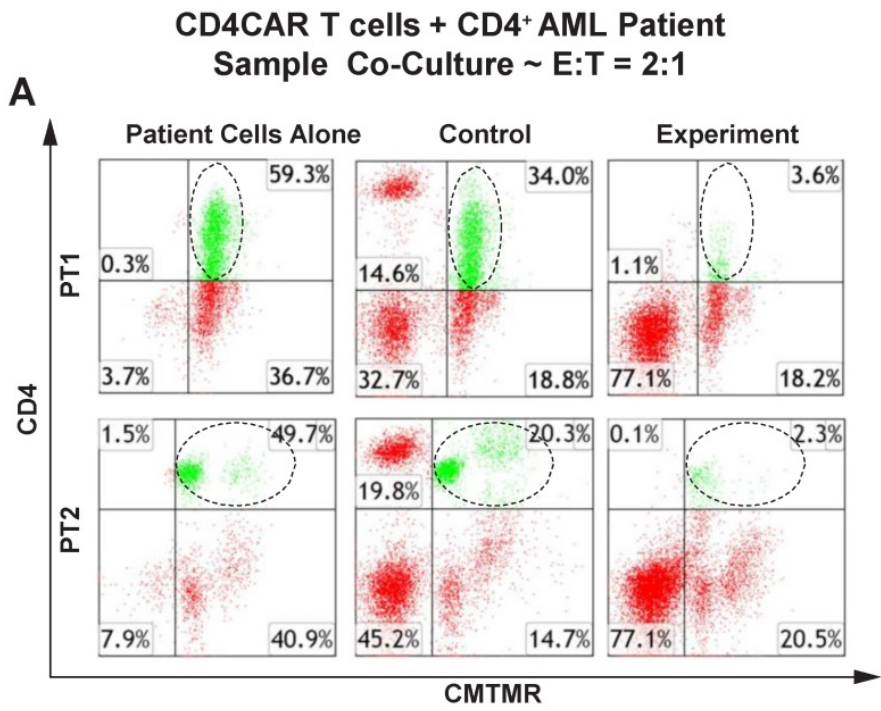

B

\section{CD4CAR T cells exhibit potent anti-leukemic effects in a systemic AML mouse model in vivo}

To investigate the anti-leukemic activity of CD4CAR T cells in vivo, we established a xenogeneic mouse model bearing the leukemic MOLM-13 cell line. Mice were given a single dose of CD4CAR T cells or GFP control $\mathrm{T}$ cells and tumor burden was measured on day 3, 6, and 11 (Figure 4A, 4B). By day 6, CD4CAR $\mathrm{T}$ cells exhibited profound leukemia suppression, indicated as a $77 \%$ reduction of leukemic burden in the CAR $\mathrm{T}$ cell treated group, compared with the GFP control $\mathrm{T}$ cell treated group. This capacity of CD4CAR $\mathrm{T}$ cells to control leukemia progression was enhanced by day 11, as the reduction rate reached $96 \%$ compared with the GFP control T cell group (Figure 4C). Furthermore, the survival time of CD4CAR $\mathrm{T}$ cell treated mice was significantly extended compared to that of GFP control $\mathrm{T}$ cell treated mice $(P=0.0002$, Figure $4 \mathrm{D})$, which indicated an efficient repression of the leukemia by CD4CAR T cells in vivo.

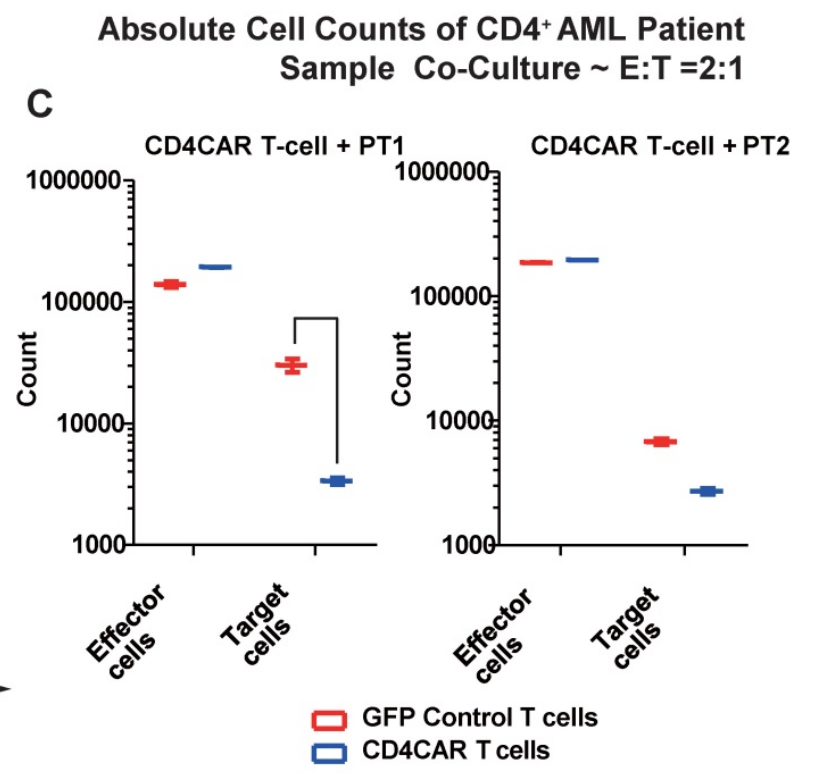

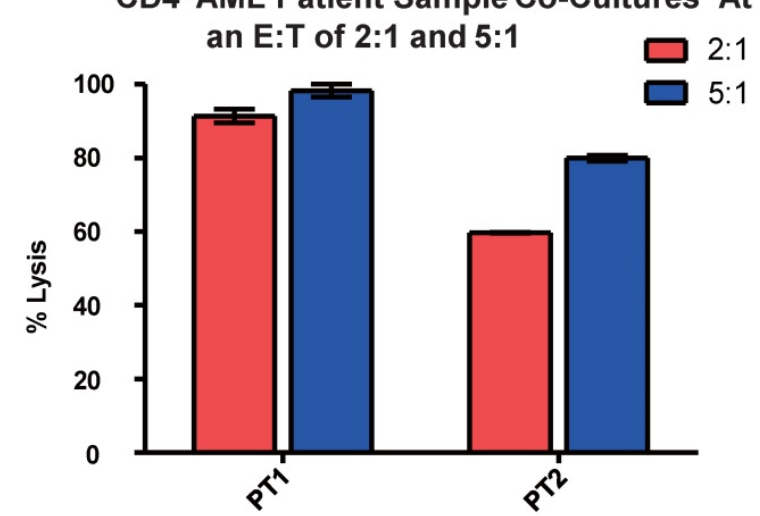

Figure 3: CD4CAR T-cells ablate CD4+ AML patient cells in vitro. (A) Co-culture experiments with CD4CAR T-cells were performed at E: T ratios of 2:1 and 5:1 for 24 hours. Target cells used are derived from Patient 1 (PT1) and Patient 2 (PT2). Target populations quantified with flow cytometry using CD3 and pre-labeled CMTMR target cells to distinguish T-cell and target cell populations. Populations encircled highlight target cell lysis. (B) Graphical summary of CD4CAR T-cell in vitro assays against AML patient cells. Each bar represents the average percent cell lysis for duplicate samples; $N=2$ for all. (C) Absolute cell counts of target cell cultures with CD4CAR T-cells at an E: T ratio of 2:1. Control and CD4CAR treatment samples are labeled in red and blue respectively with effector and target cell counts performed via FACS analysis. 
A

Day 3

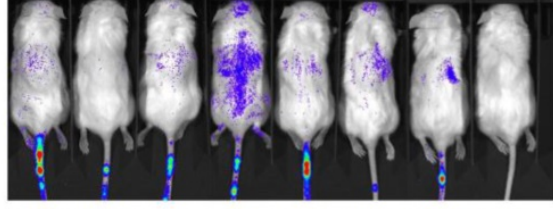

Day 6

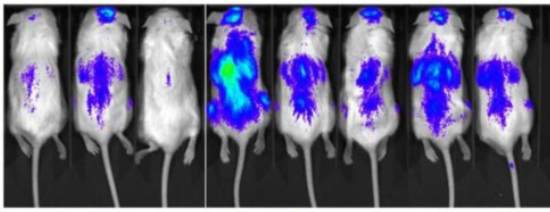

Day 11

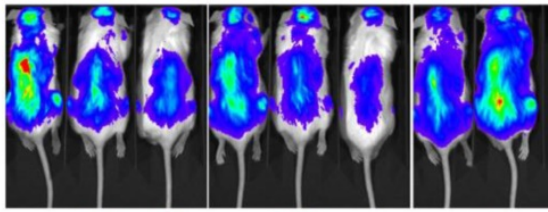

Day 15

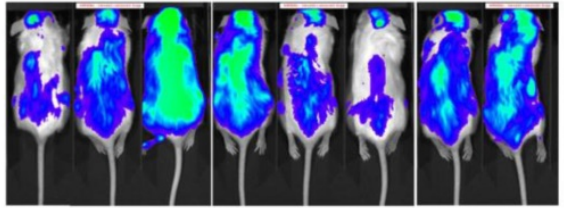

CD4CAR T cells control CD4+ MOML13 Leukemia growth in vivo

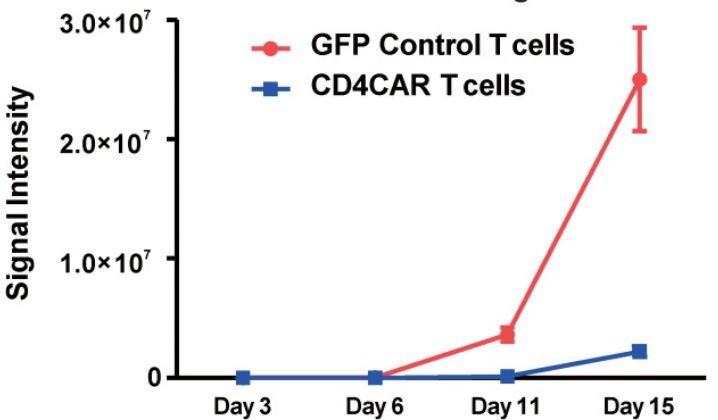

D

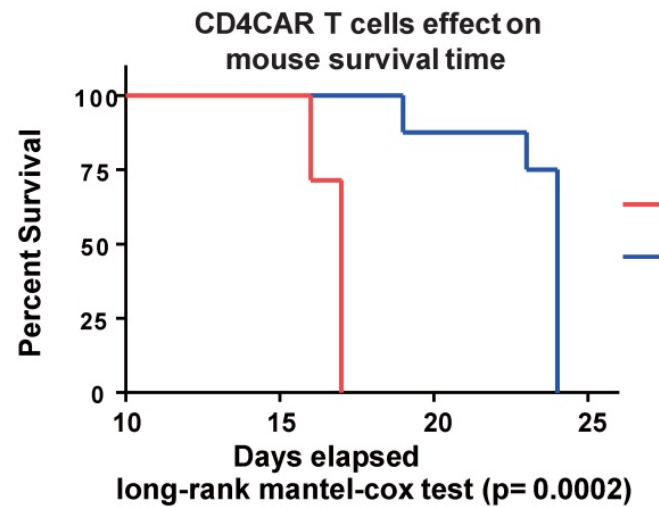

CD4CAR T Cells
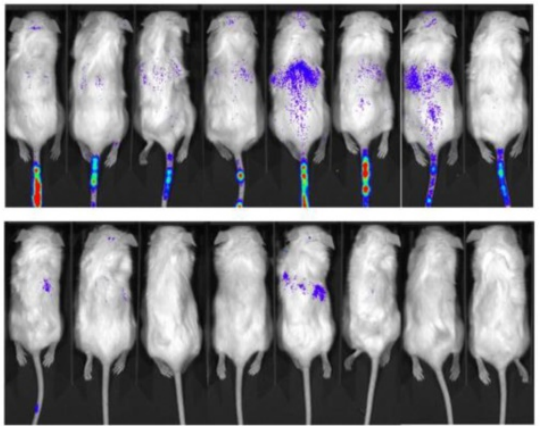

$1.0 \times 10 \mathrm{e} 4$

$8.0 \times 10 e 5$

$8.0 \times 10 \mathrm{e} 4$

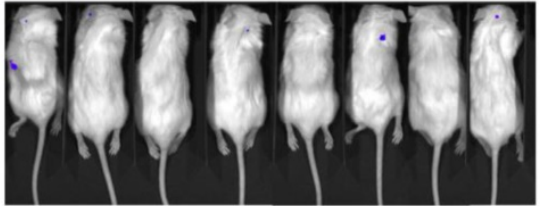

$3.0 \times 10 \mathrm{e} 7$

$3.0 \times 10 \mathrm{e} 6$

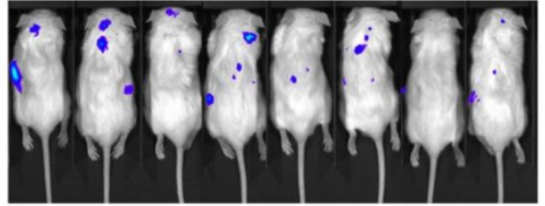

$2.0 \times 10 e 8$

$2.0 \times 10 \mathrm{e} 7$
C CD4CAR T cells treatment reduces leukemia burden

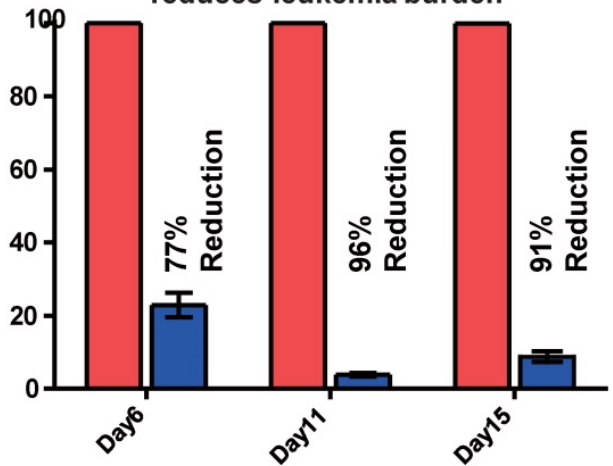

GFP Control T cells CD4CAR T cells

\section{GFP Control T cells $(n=7)$ \\ CD4CAR T cells $(n=8)$}

Figure 4: CD4CAR T-cells demonstrate profound anti-leukemic effects in vivo. (A) Elimination of luciferase-expressing MOLM-13 cells in xenografted mice treated with CD4CAR T-cells as measured via IVIS imaging. Average flux of IVIS imaging was measured and compared in the CD4CAR T-cell treated mice (right, $\mathrm{N}=8$ ) versus that in the control T-cells treated mice (left, $\mathrm{N}=8$ ) on day 3, 6, and 11. The percent cell lysis by CD4CAR T-cells relative to control was determined via luciferin signal. (B) CD4CAR T-cells control MOLM-13 tumor growth in vivo. Average light intensity (in photons per second) measured for the CD4CAR T-cell injected mice was compared to that of GFP control $\mathrm{T}$-cell injected mice. P-values are indicated at specific time-points, demonstrating a statistically significant reduction in the relative tumor burden by CD4CAR T-cells as compared to GFP control on days $6(\mathrm{P}=.00174)$ and $11(\mathrm{p}=<.0001)$. (C) CD4CAR T-cells reduce MOLM-13 tumor burden in vivo. Percent luciferin signal measured for CD4CAR T-cell injected mice and demonstrated as percent difference in signal from GFP control T-cell injected mice. The percent reduction of tumor burden on days 6 and 11 is shown. (D) CD4CAR T-cell treated mice survive significantly longer than control mice. Kaplan-Meier survival curve for CD4CAR T-cell treated mice compared to GFP control treated mice (log-rank (Mantel-Cox) test p-value $=.0002$ ). On Day 24, all CD4CAR-treated mice were sacrificed for persistency studies. 


\section{Generation and enrichment of CD4CAR NK cells}

In this study, we also developed CD4CAR NK cells to target AML. CD4CAR NK cells were transduced as previously described 22, 23, with a transduction efficiency of $16 \%$ as determined by flow cytometry. In order to further enrich for CD4CAR-positive NK cells, NK cells which expressed high levels of the construct were selected for by fluorescence activated cell sorting (FACS). The enriched CD4CAR NK cells were expanded in vitro and exhibited a stable expression rate over $85 \%$ (Figure 1C).

\section{CD4CAR NK cells robustly eliminate CD4-expressing AML cell lines}

We next evaluated the anti-leukemic activity of CD4CAR NK cells using CD4-positive AML cell lines: THP-1, U937, and MOLM-13. We co-cultured THP-1, U937, or MOLM-13 cells with CD4CAR NK cells for 24 hours at E: $\mathrm{T}$ ratios of 2:1 and 5:1 (Figure 5A). Compared with GFP control NK cells, we observed that CD4CAR NK cells consistently and robustly eliminated all CD4-positive leukemic cells (Figure 5A, 5B). At an E:T ratio of 2:1, we found that that CD4CAR NK cells already demonstrated significant cytotoxicity, and were able to lyse $89 \%, 98 \%$, and $77 \%$ of tumor cells in co-culture with THP-1, U937, and MOLM-13 cells, respectively. When the $\mathrm{E}: \mathrm{T}$ ratio increased to $5: 1$, nearly $100 \%$ lysis was observed in all three cell lines (Figure 5C).
A CD4CAR NK cells + AML Leukemic Cell Line Co-Culture E:T = 2:1

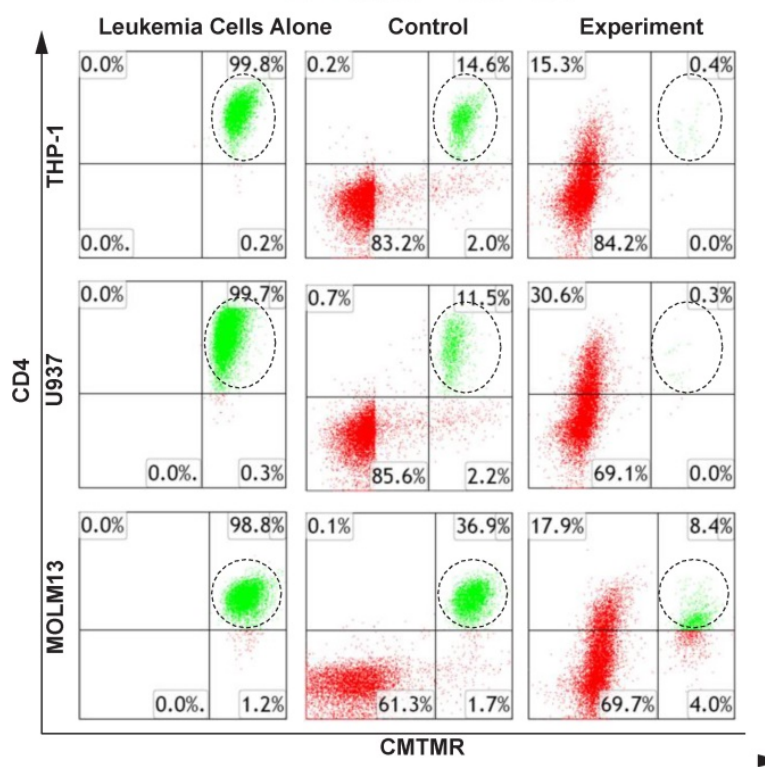

C
AML Leukemic Cell Line Co-Cultures At an $E: T$ of $2: 1$ and $5: 1$

\section{B Absolute Cell Counts of AML Cell Line Co- Culture $\sim \mathrm{E}: \mathrm{T}=2: 1$}

CD4CAR NK cells + THP-1 CD4CAR NK cells + U937
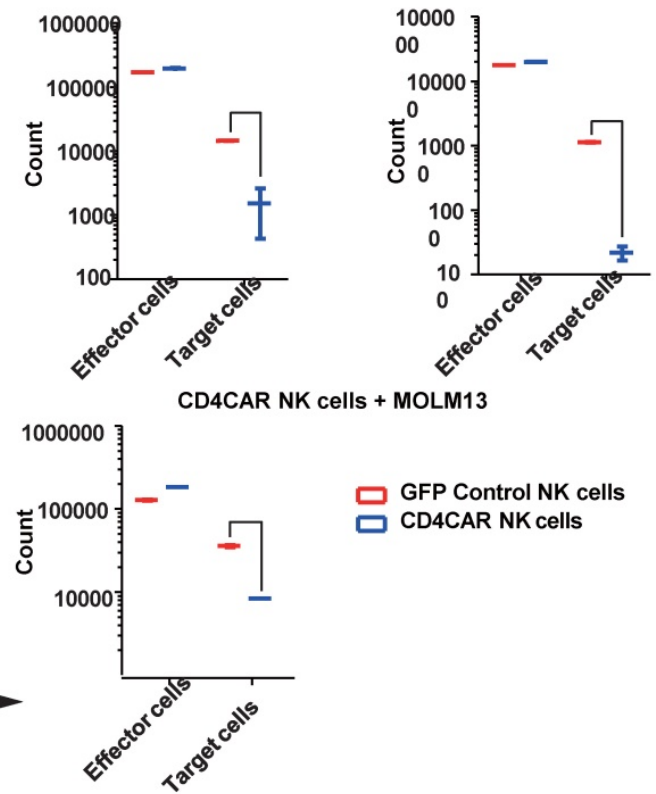

GFP Control NK cells $\square$ CD4CAR NK cells

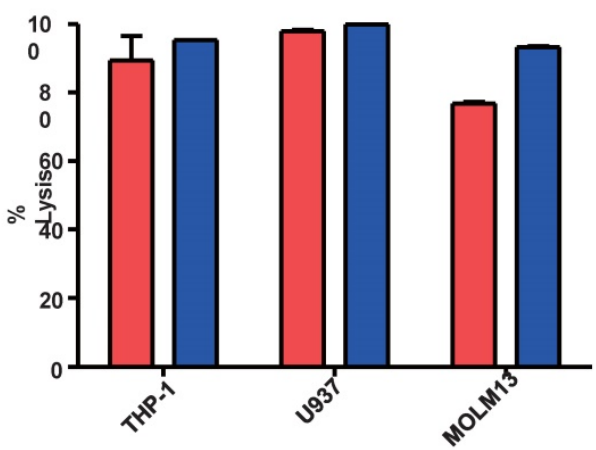

2:1

$\square$ 5:1

Figure 5. CD4CAR NK-92 cells ablate CD4+ AML cell lines in vitro. (A) Co-culture experiments with CD4CAR NK-92 cells were performed at E: T ratios of 2:1 and 5:1 for 24 hours. CD4+ target cell lines used are THP-1, U937, and MOLM13. Target populations were quantified with flow cytometry using CD4 and pre-labeled CMTMR target cells to distinguish NK and target cell populations. Populations encircled highlight target cell lysis. (B) Graphical summary of CD4CAR NK-92 cell in vitro assays against AML cell lines. Each bar represents the average percent cell lysis for duplicate samples; $N=2$ for all. (C) Absolute cell counts of target cell cultures with CD4CAR NK-92 cells at an E: T ratio of 2:1. Control and CD4CAR treatment samples are labeled in red and blue respectively with effector and target cell counts performed via FACS analysis. 


\section{CD4CAR NK cells specifically target and lyse CD4+ primary AML leukemic cells}

We then verified the efficiency of CD4CAR NK cells in specifically recognizing and eliminating CD4-positive primary leukemic cells from AML patient bone marrow samples. Again, two patient samples PT1 and PT2 were co-cultured with CD4CAR NK cells at E: T ratios of 2:1 and 5:1 (Figure 7A). A robust lysis rate was already observed at $\mathrm{E}$ : $\mathrm{T}$ ratio of $2: 1$, and the lysis rate further approached $80-100 \%$ when the E: T ratio increased to 5:1 (Figure 6A, 6C). The result of absolute cell counts also confirmed a significant decrease of the target leukemic cells after co-culture with CD4CAR NK cells (Figure 6B).

A CD4CAR NK cells + CD4 $4^{+}$AML Patient Sample Co-Culture $\sim \mathrm{E}: \mathrm{T}=2: 1$

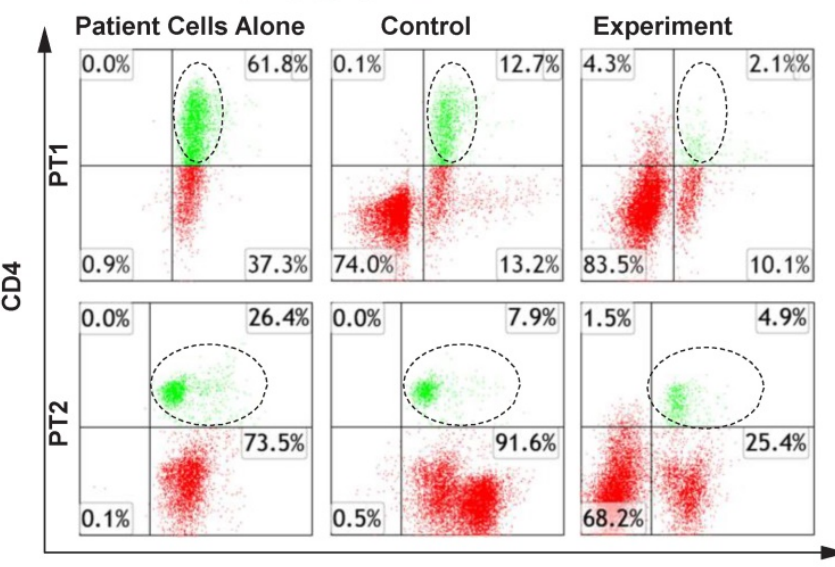

CMTMR

C

CD4+ AML Patient Sample Co-Cultures At an $E: T$ of $2: 1$ and $5: 1$

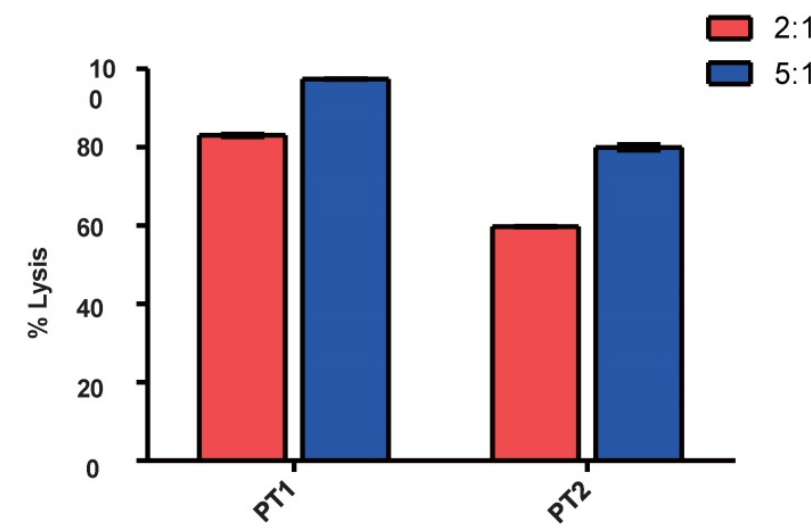

CD4CAR NK cells exhibit potent anti-leukemic effects in a systemic AML mouse model in vivo

To further model in vivo activity, we examined the anti-leukemic effects of CD4CAR NK cells in a MOLM-13 leukemic mouse model. Mice were treated with either CD4CAR NK cells or GFP control NK cells and tumor burden was measured on days 3, 7, and 9 (Figure 7A). CD4CAR NK cells efficiently suppressed leukemic growth, with a $90 \%$ tumor reduction by day 7 compared with control NK cells. This difference increased to $98 \%$ by day 9 (Figure 7B, 7C). To maintain the NK cell population, a second treatment dose was given on day 10 . This resulted in a significantly prolonged survival time in CAR NK cell treated mice than in control NK cell treated mice $(\mathrm{P}=0.0017$, Figure 7D).

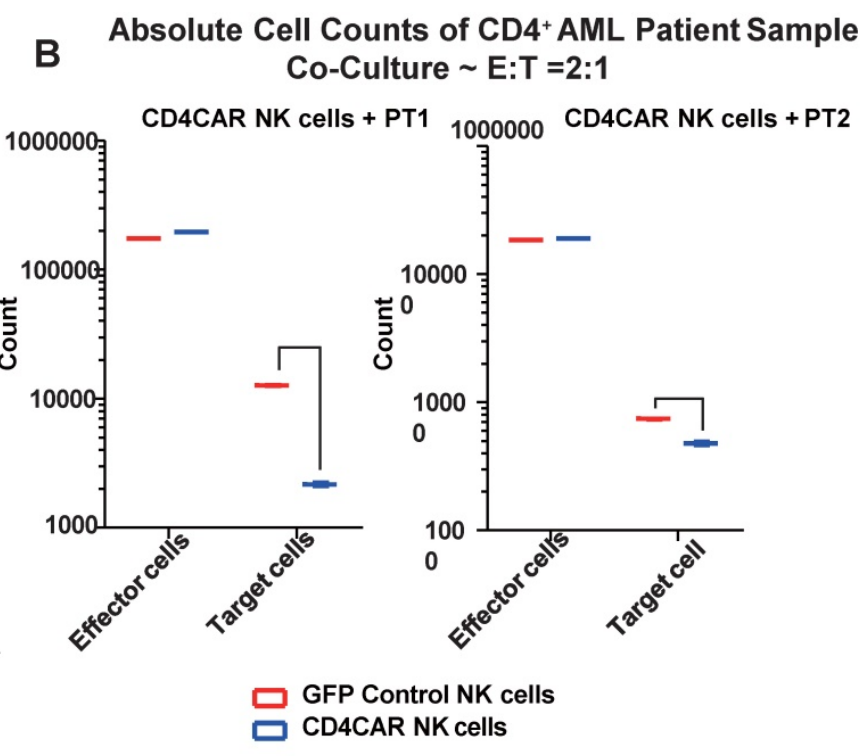

Figure 6. CD4CAR NK-92 cells ablate CD4+ AML patient cells in vitro. (A) Co-culture experiments with CD4CAR NK-92 cells were performed at an effector to target ratio of 2:1 and 5:1 for 24 hours. Target cells used are derived from Patient 1 (PT1) and Patient 2 (PT2). Target populations were quantified with flow cytometry using CD4 and pre-labeled CMTMR target cells to distinguish NK and target cell populations. Populations encircled highlight target cell lysis. (B) Graphical summary of CD4CAR NK-92 cell in vitro assays against AML patient cells. Each bar represents the average percent cell lysis for duplicate samples; $N=2$ for all. (C) Absolute cell counts of target cell cultures with CD4CAR NK-92 cells at E: T ratios of 2:1. Control and CD4CAR treatment samples are labeled in red and blue respectively with effector and target cell counts performed via FACS analysis. 
A IVIS Imaging of MOLM13-Luc+ Xenografted Mouse Model \#2 CD4CAR NK Cells Efficacy in vivo GFP Control NK-Cells CD4CAR NK-Cells

Day 3
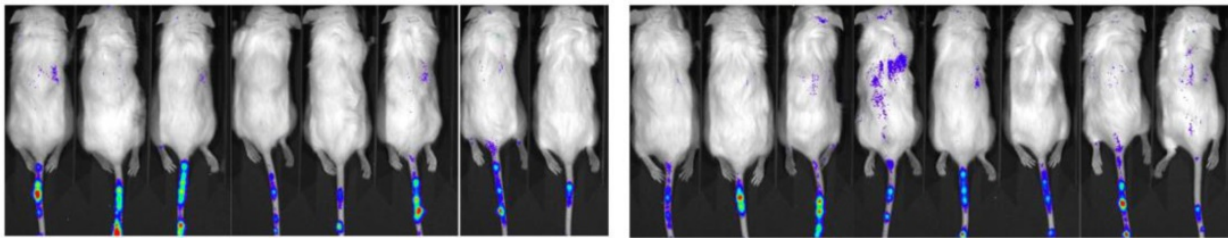

$2.0 \times 10 \mathrm{e} 5$

Day 7
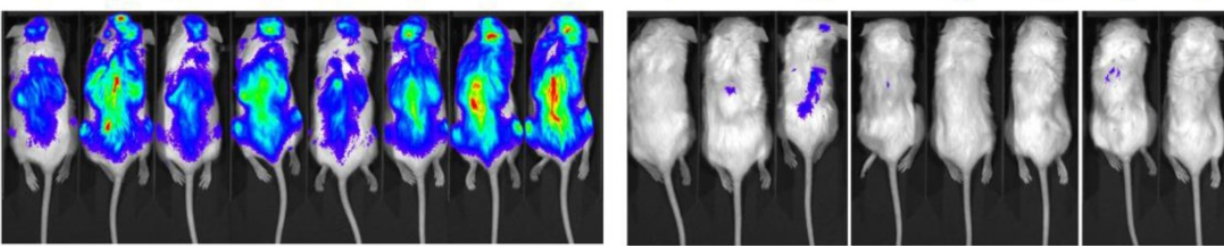

$2.0 \times 10 \mathrm{e} 4$
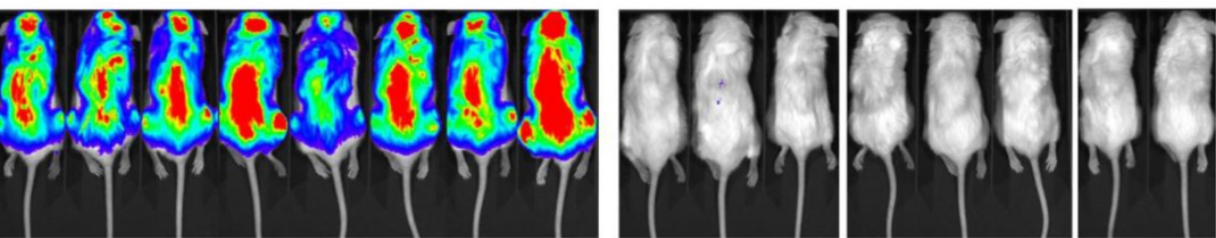

$2.0 \times 10 \mathrm{e} 5$

$1.0 \times 10 \mathrm{e} 7$

Day 9

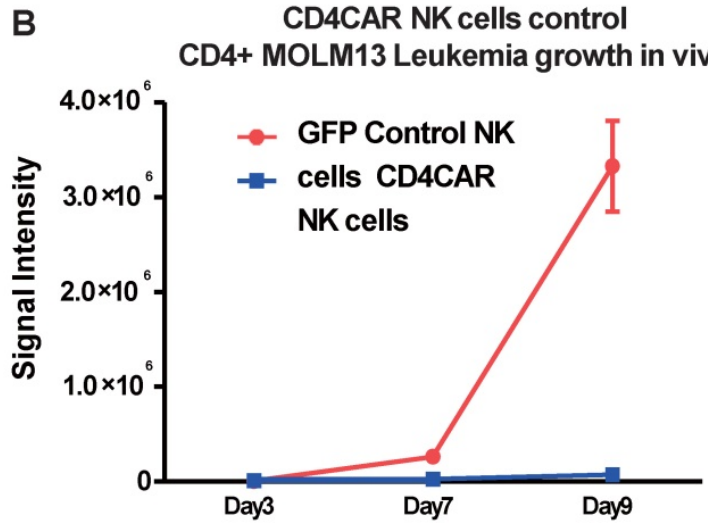
C
CD4CAR NK-cell treatment reduces leukemia burden

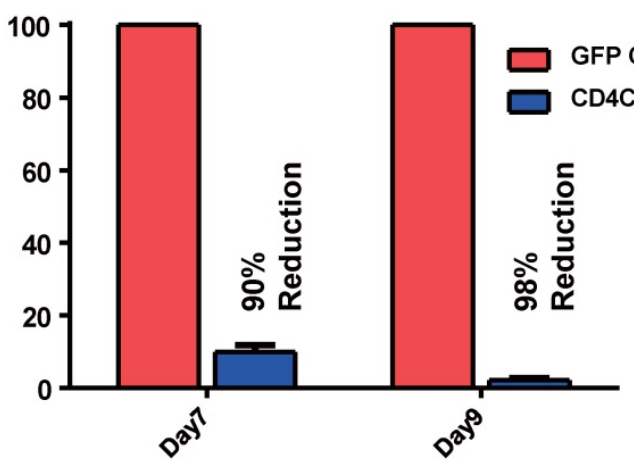
D
CD4CAR NK cells effecton mouse survival time

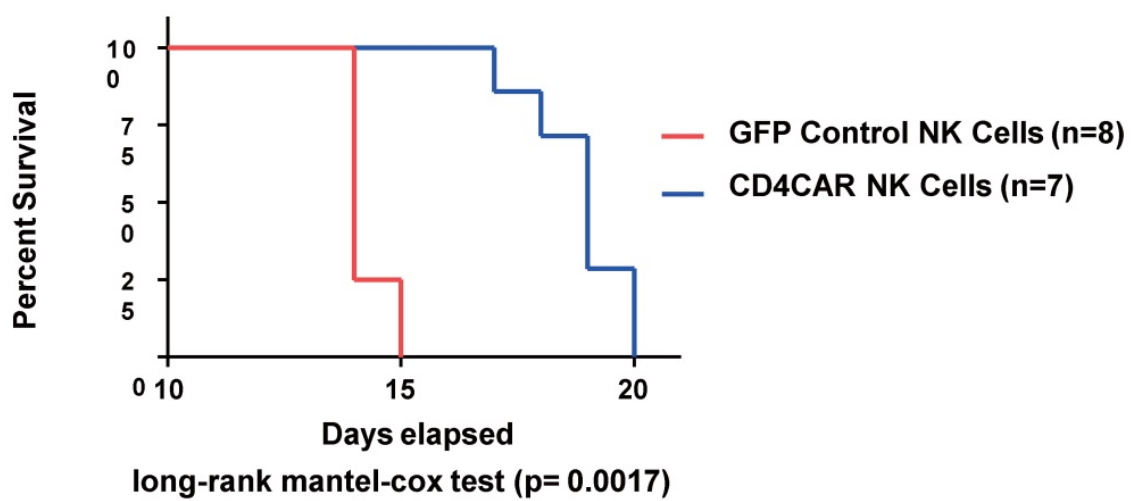

Figure 7: CD4CAR NK-92 cells demonstrate profound anti-leukemic effects in vivo. (A) Elimination of luciferase-expressing MOLM-13 cells in xenografted mice treated with CD4CAR NK-cells as measured via IVIS imaging. Average flux of IVIS imaging was measured and compared in the CD4CAR NK-cell treated mice (right, N=8) versus that in the control NK cells treated mice (left, $N=8$ ) on days 3,7 , and 9 . The \% cell lysis by CD4CAR T-cells relative to control was determined via luciferin signal. (B) CD4CAR NK-cells control MOLM-13 tumor growth in vivo. Average light intensity (in photons per second) measured for the CD4CAR NK cell injected mice was compared to that of GFP control NK-cell injected mice. P-values are indicated at specific time-points, demonstrating a statistically significant reduction in the relative tumor burden by CD4CAR NK-92 cells as compared to GFP control on days $7(\mathrm{p}=<.00001)$ and $9(\mathrm{p}=<.00001)$. (C) CD4CAR NK cells reduce MOLM-13 tumor burden in vivo. Percent luciferin signal was measured for CD4CAR T-cell injected mice and demonstrated as percent difference in signal from GFP control T-cell injected mice. The percent reduction of tumor burden on days 7 and 9 is shown. (D) CD4CAR NK cell treated mice survive significantly longer than control mice. Kaplan-Meier survival curve for CD4CAR NK cell treated mice compared to GFP control treated mice (log-rank (Mantel-Cox) test p-values $=0.0017$ ). On Day 24, all CD4CAR-treated mice were sacrificed for persistency studies. 


\section{Discussion}

The "on-target off-tumor" myelotoxicity remains a challenge in carrying over the success of targeted therapy from lymphoid leukemias to myeloid targeting because myeloid antigens are likely shared on the hematopoietic stem cells 2, 3, 5, 6. We previously showed that our CD4CAR did not affect the CD34+ cord blood granulocyte/macrophage or erythroid colony formation (CFU) ex vivo, which indicates that HSC ability to populate the marrow is not affected by CD4CAR treatment ${ }^{10}$. Our novel approach using CD4-directed therapy for AML is an alternative way to target an antigen that is not ubiquitously expressed on hematopoietic stem cell or non-hematopoietic cells, therefore circumventing potential relevant toxicities. The use of CD4, traditionally thought of as a lymphoid marker, to target a myeloid malignancy represents an innovative therapy that can supplement current AML available treatments. We used two effector systems, T-cells and NK cells, to implement CD4CAR therapy, creating novel treatment options for relapsed and refractory patients where none previously existed. We showed that CD4CAR-engineered $\mathrm{T}$ cells and NK cells specifically killed CD4+ malignant AML cell lines and primary AML patients' blasts in vitro. Furthermore, these CD4CAR-redirected effector cells exhibited profound killing capacity against CD4-positive AML cells in vivo, and efficiently controlled leukemia progression and prolonged survival in xenograft mouse models.

CD4 is expressed on a significant number of AML patients. $65.0 \%$ and $78.3 \%$ of M4 and M5 subtypes, respectively and in $30-40 \%$ of the other AML subtypes express this antigen on blasts cells ${ }^{10}$. In our experience, when positive, CD4 expression corresponded with the blast percentage (data not shown). CD4CAR T cell therapy for CD4+ AML has the potential to reduce leukemic burden or to induce remission in refractory cases in preparation and as a bridge to definitive hematopoietic stem cell transplantation. Conversely, shorter lived CAR NK cells last only around 2 weeks, enabling a "hit and run" approach ${ }^{24}$ and the potential to accomplish both of these treatment goals. NK cells also offer additional CAR-independent, granule-mediated killing strategies via antibody-dependent cell-mediated cytotoxicity (ADCC) and death receptor pathways 25 , 26. CAR NK cells also function as "serial killers", destroying multiple target cells in succession 27 . Together, these properties of CAR NK cells make them particularly suited for a rapid, yet transient reduction in tumor burden. Moreover, CAR NK cells pose little or even no risk of graft-versus-host disease
(GvHD) reaction, and do not release cytokine-storm inducing IL-6, the greatest risk in CAR T cell therapy 28 . For patients who are unfit for more intensive therapy using CAR T cell therapy, the shorter lifespan and reduced toxicity of CAR NK cells may serve as a palliative treatment and eliminate concerns of long-term side effects as seen in CAR $\mathrm{T}$ cells and potentially qualifying these patients for curative SCT utilizing reduced conditioning. Additionally and because of their short life NK CAR cells might potentially be given more than once if needed.

Our studies found that CD4CAR-redirected immune effector cells efficiently control leukemia progress in vitro and in vivo. Irrespective of a CD4-depleted environment, CD8+ T cells alone are likely enough to eliminate tumors. A recent report showed that infusion of CD8+ CAR-modified T cells was sufficient to maintain long-term target cell eradication and leukemia remission in a mouse model 29. Recent studies also showed that memory CD8+ cells or less-differentiated cell subsets were associated with superior $\mathrm{T}$ cell engraftment, persistence, and antitumor immunity 30,31. Notably, our CD4CAR T cells had a predominant cell population with a central memory-like phenotype (CD8+CD45RO+CD62L+) at the end of each culture, as reported in our previous study. Additionally, CD4CAR therapy might provide an added benefit of depleting CD4+ $\mathrm{T}$ cells and modulating the tumor microenvironment. CD4-positive cells include Tregs, Th2 cells, Tr1/3 cells, a subpopulation of myeloid-derived suppressor cells (MDSCs), and dendritic cells (DCs) 33,34,35. Recent studies have shown that increased frequency of Treg cell number correlates to cancer progression, and that Tregs are important immunosuppressive factors in the AML microenvironmen ${ }^{36,37}$. Tregs limit CD8+ effector cell differentiation and NK cell cytotoxicity (Pedroza-Pacheco 2013) ${ }^{38}$ and impair the efficacy of adoptive T-cell therapy ${ }^{39}$.

While transient CD4+ lymphodepletion remains a valid concern together with the "on-target off-tumor" toxicities associated with immunotherapy. We envision that treatment with the CD4CAR will be most helpful and best applied to reduce tumor burden or induce remission in preparation "as a bridge" to more definitive therapy with hematopoietic stem cell transplantation. This approach will further minimize long term lymphodepletion via elimination of the CAR cells by pre-transplant conditioning regimens and consequently via a whole new allogeneic lymphoid engraftment. Utilization of CD4CAR in these otherwise refractory patients will provide a potentially curative treatment if they successfully qualify for SCT and again, without significant concerns about long term effects of CAR persistence 
and associated lymphodepletion. As a further precaution against off-target cytotoxicities and CD4+ lymphodepletion especially for those patients who cannot make it to transplant, alemtuzumab-based safety switch is expected to allow fast and thorough pharmacologic ablation of these cells. Alemtuzumab could eliminate not only peripheral blood CAR T cells, but also tissue-infiltrating CAR $\mathrm{T}$ cells making CD4CAR safe to develop for clinical use, supplementary FIG 2S. Furthermore, in this study we also present the option of utilizing NK CAR that is typically associated with production of a different spectrum of cytokines compared to T cells including $\gamma$-Interferon, IL-3 and the granulocyte macrophage colony stimulating factor and also is with much shorter survival without compromising its malignant cell killing efficiency ${ }^{40,41}$.

\section{Conclusions}

CD4 is expressed in a significant proportion of AML patients. CD4CAR represents a novel approach to specifically and robustly target malignant CD4+ AML blasts. Effective remission induction or disease burden reduction is direly needed in preparation for allogeneic stem cell transplant in refractory AML patients. CD4CAR is expected to be effective and safe in this setting. Post-transplant engrafted $T$ cells will rescue $\mathrm{CD} 4$ positive lymphodepletion after CD4CAR treatment and hence offset longer term immune suppression. Adoption of CAR technology in both $\mathrm{T}$ cells and NK cells provides versatility and greater therapeutic range for patients who previously had no options. CD4CAR T cell and NK cell therapy are effective methods for augmenting current AML therapies and their prompt application in clinical trials is warranted.

\section{Supplementary Material}

Supplementary figures.

http://www.jcancer.org/v10p4408s1.pdf

\section{Abbreviations}

CD4CAR: CD4 Chimeric Antigen Receptor directed T cells; AML: Acute Myeloid Leukemia; HSCs: Hematopoietic Stem Cells; CAR-T cells: Chimeric Antigen Receptor T cells; NK: Natural killer.

\section{Author Contribution}

HS: Corresponding author. Participated in experimental design, analyzed data and wrote the paper. KP: second first author, designed, supervised and performed in vivo experiments and contributed to writing the paper. Masayuki Wada, Xiao Shuai, Lulu E. Yan, Jessica C. Petrov: Performed the experimental work. Yupo Ma: funded and supervised this work and senior author. All authors read and approved the final manuscript.

\section{Competing Interests}

KGP, MW and YM are co-inventors of this technology and hold the patents related to the contents of this manuscript, and are employees of iCell Gene Therapeutics, LLC.

\section{References}

1. Maude SL, Teachey DT, Porter DL, Grupp SA. CD19-targeted chimeric antigen receptor T-cell therapy for acute lymphoblastic leukemia. Blood. 2015;125(26):4017-4023.

2. Mardiros A, Dos Santos C, McDonald T, BrownC E, Wang X, Budde L E, et al. $\mathrm{T}$ cells expressing CD123-specific chimeric antigen receptors exhibit specific cytolytic effector functions and antitumor effects against human acute myeloid leukemia. Blood. 2013;122(18):3138-3148.

3. Gill S, Tasian SK, Ruella M, et al. Preclinical targeting of human acute myeloid leukemia and myeloablation using chimeric antigen receptor-modified T cells. Blood. 2014;123(15):2343-2354.

4. Dutour A, Marin V, Pizzitola I, et al. In Vitro and In Vivo Antitumor Effect of Anti-CD33 Chimeric Receptor-Expressing EBV-CTL against CD33 Acute Myeloid Leukemia. Adv Hematol. 2012;2012:683065.

5. Kenderian SS, Ruella M, Shestova O, et al. CD33-specific chimeric antigen receptor $\mathrm{T}$ cells exhibit potent preclinical activity against human acute myeloid leukemia. Leukemia. 2015;29(8):1637-1647.

6. Casucci M, Nicolis di Robilant B, Falcone L, Camisa B. Norelli M.Genovese P. et al. CD44v6-targeted T cells mediate potent antitumor effects against acute myeloid leukemia and multiple myeloma. Blood. 2013;122(20):3461-3472.

7. Lynn RC, Poussin M, Kalota A, Feng Y, Low P, Dimitrov D S, Powell D. J.Jr.. Targeting of folate receptor beta on acute myeloid leukemia blasts with chimeric antigen receptor-expressing T cells. Blood. 2015;125(22):3466-3476.

8. Ritchie DS, Neeson PJ, Khot A, Peinert S, Tai T, Tainton K et al. Persistence and efficacy of second generation CAR T cell against the LeY antigen in acute myeloid leukemia. Mol Ther. 2013;21(11):2122-2129.

9. Wang QS, Wang Y, Lv HY, Han Q, Fan H, Guo B et al. Treatment of CD33-directed chimeric antigen receptor-modified $\mathrm{T}$ cells in one patient with relapsed and refractory acute myeloid leukemia. Mol Ther. 2015;23(1):184-191.

10. Miwa H, Mizutani M, Mahmud N, Yamaguchi M, Takahashi T, Shikami M et al. Biphasic expression of CD4 in acute myelocytic leukemia (AML) cells: AML of monocyte origin and hematopoietic precursor cell origin. Leukemia. 1998;12(1):44-51.

11. Pinz KG, Yakaboski E, Jares A, Liu H, Firor AE, Chen KH, Wada M, Salman H, Tse W, Hagag N, Lan F, Leung $E$, Jiang $X$, and Ma Y. Targeting T-cell malignancies using anti-CD4 CAR NK-92 cells. Oncotarget. 2017; (8):112783-112796.

12. Scheerens $\mathrm{H}, \mathrm{Su} \mathrm{Z}$, Irving $\mathrm{B}$, et al. MTRX1011A, a humanized anti-CD4 monoclonal antibody, in the treatment of patients with rheumatoid arthritis: a phase I randomized, double-blind, placebo-controlled study incorporating pharmacodynamic biomarker assessments. Arthritis Res Ther. 2011;13(5):R177.

13. Moreland LW, Pratt PW, Bucy RP, Jackson BS, Feldman JW, Koopman WJ. Treatment of refractory rheumatoid arthritis with a chimeric anti-CD4 monoclonal antibody. Long-term followup of CD4+ T cell counts. Arthritis Rheum. 1994;37(6):834-838.

14. Prinz JC, Meurer M, Reiter C, Rieber EP, Plewig G, Riethmuller G. Treatment of severe cutaneous lupus erythematosus with a chimeric CD4 monoclonal antibody, cM-T412. J Am Acad Dermatol. 1996;34(2 Pt 1):244-252.

15. Lindsey JW, Hodgkinson S, Mehta R, Siegel R, C.Mitchell D J, Lim M et al. Phase 1 clinical trial of chimeric monoclonal anti-CD4 antibody in multiple sclerosis. Neurology. 1994;44(3 Pt 1):413-419.

16. Knox S1, Hoppe RT, Maloney D, Gibbs I, Fowler S, Marquez C, Cornbleet PI, Levy R. Treatment of cutaneous T-cell lymphoma with chimeric anti-CD4 monoclonal antibody. Blood. 1996 Feb 1;87(3):893-9.

17. Alsamah W, Romia Y. Modification of natural killer cells to target tumors. Int. J. Pharm. Clin. Res. 2014;6: 97-100.

18. Altvater B., Landmeier S., Pscherer S., Temme J., Schweer K., Kailayangiri S., et al. 2B4 (CD244) signaling by recombinant antigen-specific chimeric receptors costimulates natural killer cell activation to leukemia and neuroblastoma cells. Clin. Cancer Res. 2009;15:4857-4866 10.1158/1078-0432.

19. Chu J., Deng Y., Benson D. M., He S., Hughes T., Zhang J., et al. CS1-specific chimeric antigen receptor (CAR)-engineered natural killer cells enhance in vitro and in vivo antitumor activity against human multiple myeloma. Leukemia 2014;28:917-927.

20. Imai C., Iwamoto S., Campana D. (). Genetic modification of primary natural killer cells overcomes inhibitory signals and induces specific killing of leukemic cells. Blood 2005;106:376-383.

21. Kruschinski A., Moosmann A., Poschke I., Norell H., Chmielewski M., Seliger B., et al. (). Engineering antigen-specific primary human NK cells against HER-2 positive carcinomas. Proc. Natl. Acad. Sci. U.S.A. 2008;105:17481-17486 
22. Chen $\mathrm{KH}$, Wada M, Firor AE, et al. Novel anti-CD3 chimeric antigen receptor targeting of aggressive T cell malignancies. Oncotarget. 2016;7(35):56219-56232.

23. Chen KH, Wada M, Pinz KG, et al. Preclinical targeting of aggressive T-cell malignancies using anti-CD5 chimeric antigen receptor. Leukemia. 2017.

24. Glienke W, Esser R, Priesner C, et al. Advantages and applications of CAR-expressing natural killer cells. Front Pharmacol. 2015;6:21.

25. Chester C, Fritsch K, Kohrt HE. Natural Killer Cell Immunomodulation: Targeting Activating, Inhibitory, and Co-stimulatory Receptor Signaling for Cancer Immunotherapy. Front Immunol. 2015;6:601.

26. Borrego F, Larrucea S, Solana R, Tarazona R. Editorial: NK Cell-Based Cancer Immunotherapy. Front Immunol. 2016;7:249.

27. Choi PJ, Mitchison TJ. Imaging burst kinetics and spatial coordination during serial killing by single natural killer cells. Proc Natl Acad Sci U S A. 2013;110(16):6488-6493.

28. Klingemann $H$. Are natural killer cells superior CAR drivers? Oncoimmunology. 2014;3:e28147.

29. Davila ML, Kloss CC, Gunset G, Sadelain M. CD19 CAR-targeted T cells induce long-term remission and B Cell Aplasia in an immunocompetent mouse model of B cell acute lymphoblastic leukemia. PLoS One. 2013;8(4):e61338

30. Maus MV, Grupp SA, Porter DL, June CH. Antibody-modified T cells: CARs take the front seat for hematologic malignancies. Blood. 2014;123(17):2625-2635.

31. Klebanoff CA, Gattinoni L, Restifo NP. Sorting through subsets: which T-cell populations mediate highly effective adoptive immunotherapy? J Immunother. 2012;35(9):651-660.

32. Pinz K, Liu H, Golightly M, Jares A, Lan F, Zieve GW et al. Preclinical targeting of human T-cell malignancies using CD4-specific chimeric antigen receptor (CAR)-engineered T cells. Leukemia. 2015.

33. Ghiringhelli F, Menard C, Terme M, et al. CD4+CD25+ regulatory T cells inhibit natural killer cell functions in a transforming growth factor-beta-dependent manner. J Exp Med. 2005;202(8):1075-1085.

34. Toka FN, Suvas S, Rouse BT. CD4+ CD25+ T cells regulate vaccine-generated primary and memory CD8+ T-cell responses against herpes simplex virus type 1. J Virol. 2004;78(23):13082-13089.

35. Ueha S, Yokochi S, Ishiwata Y, Ogiwara H, Chand K, Nakajima T et al. Robust Antitumor Effects of Combined Anti-CD4-Depleting Antibody and Anti-PD-1/PD-L1 Immune Checkpoint Antibody Treatment in Mice. Cancer Immunol Res. 2015;3(6):631-640.

36. Pedroza-Pacheco I, Madrigal A, Saudemont A. Interaction between natural killer cells and regulatory T cells: perspectives for immunotherapy. Cell Mol Immunol. 2013;10(3):222-229.

37. Ustun C, Miller JS, Munn DH, Weisdorf DJ, Blazar BR. Regulatory T cells in acute myelogenous leukemia: is it time for immunomodulation? Blood. 2011;118(19):5084-5095

38. McNally A, Hill GR, Sparwasser T, Thomas R, Steptoe RJ. CD4+CD25+ regulatory $\mathrm{T}$ cells control CD8+ T-cell effector differentiation by modulating IL-2 homeostasis. Proc Natl Acad Sci U S A. 2011;108(18):7529-7534.

39. Kofler DM, Chmielewski M, Rappl G, Hombach A, Riet T, Schmidt A et al. CD28 costimulation Impairs the efficacy of a redirected t-cell antitumor attack in the presence of regulatory $t$ cells which can be overcome by preventing Lck activation. Mol Ther. 2011;19(4):760-767

40. Huenecke S, Zimmermann SY, Kloess S, Esser R, Brinkmann A, Tramsen L, Koenig M, Erben S, Seidl C, Tonn T, Eggert A, Schramm A, Bader P, Klingebiel $\mathrm{T}$, Lehrnbecher T, Passweg JR, Soerensen J, Schwabe D, Koehl U. IL-2-driven regulation of NK cell receptors with regard to the distribution of CD16+ and CD16- subpopulations and in vivo influence after haploidentical NK cell infusion. J Immunother. 2010; 33(2):200-10.

41. Klingemann H. Are natural killer cells superior CAR drivers? Oncoimmunology. 2014; 3:e28147 